ارزيابى تحمل به تنش خشكى در برخى از زُنوتيٍٍاى گلرنگ (Carthamus tinctorius L.)

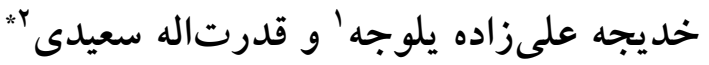

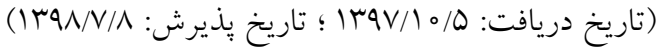

جكيده

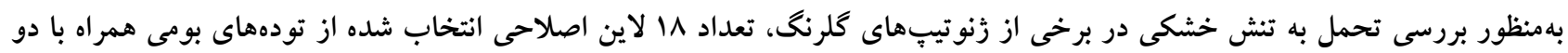

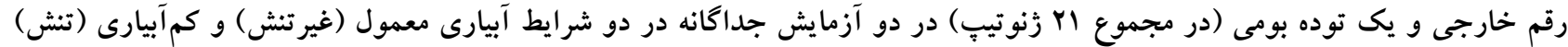

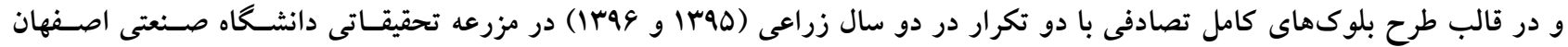

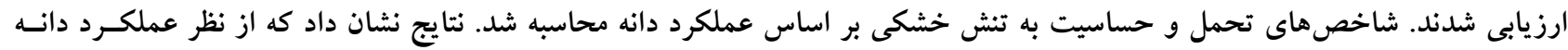

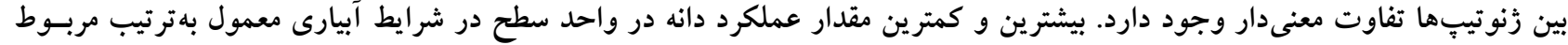

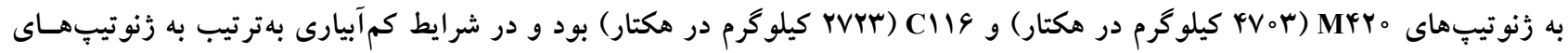

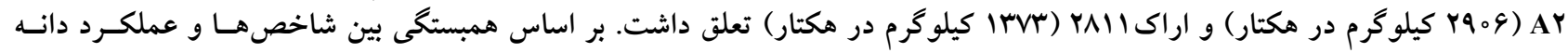

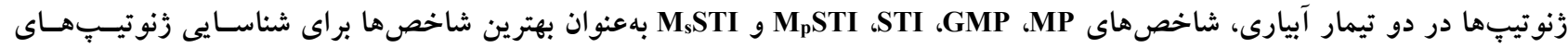

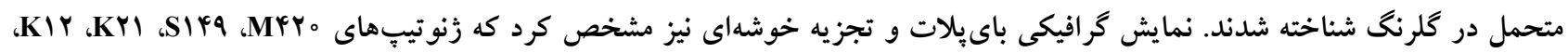

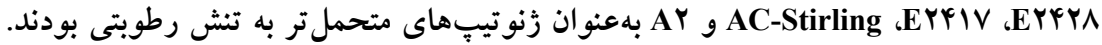

وازههاى كليدى: تنش رطوبتى، شاخصهاى تحمل، عملكرد دانه و گلرنح 
شاخص ميانخين توليد (MP) (معادلـه () نشـاندهنـده متوسط

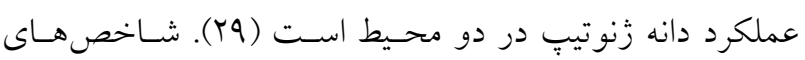

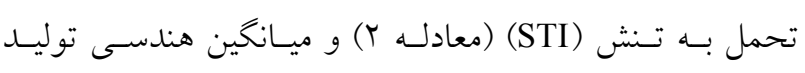

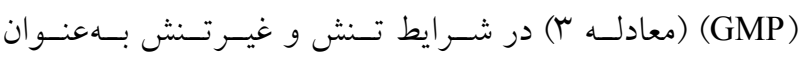

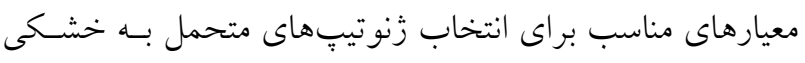

يشنهاد شدهاند (I ) (I ).

شاخصهاى ديخرى مانند شاخص مقاومت به خشكى (DI)

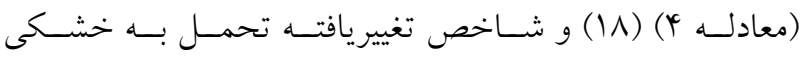

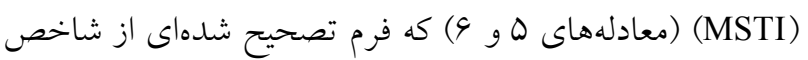

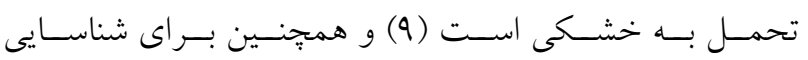

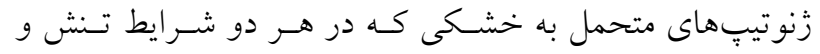
غيرتنش عملكرد مطلوب دارند، معرفى شدهاند. شاخص ميزان محصول در محيط تنش و غيـتنش (SNPI)

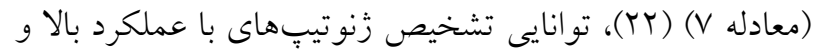

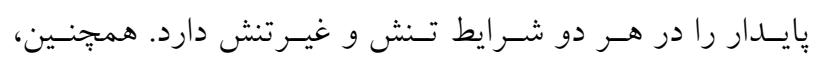

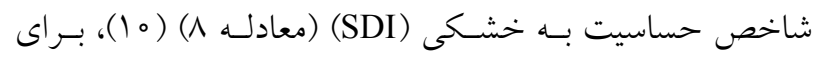
شناسايى زنوتيبه هاى متحمل بيشنهاد شده است.

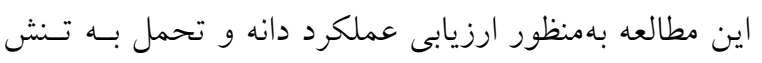

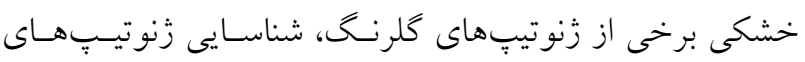

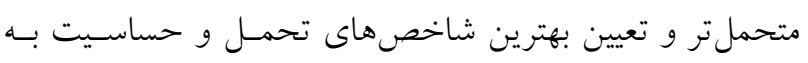
تنش خشكى انجام كرفت.

\section{مواد و روشها}

در اين تحقيق اب زنوتيبٍ شامل \1 لاين اصلاحى انتخاب شده

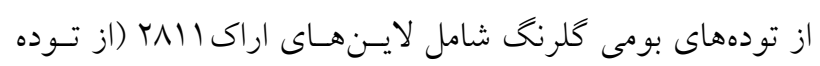

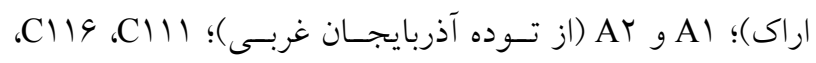

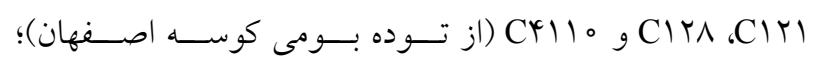

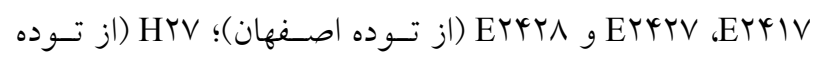

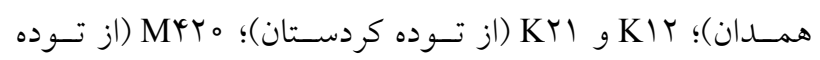

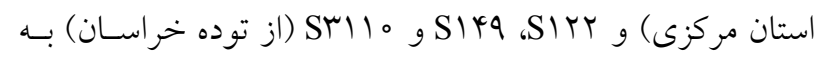

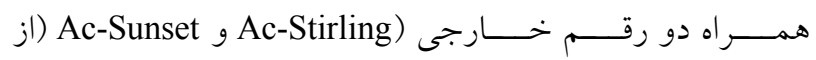
كشوركانادا)) و يك توده بومى گلرنخ اصفهان (كوسه) استفاده
كلرنغ (Carthamus tinctorius L.) يك كياه دانه روغنسى و از خانواده آستراسه است كه در سالهاى اخير بهدليل ساز گارى آن

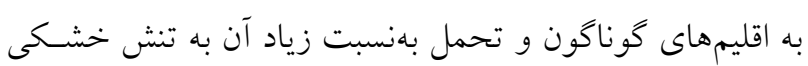

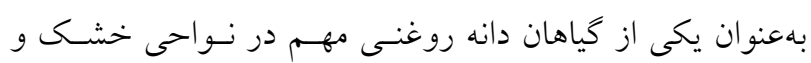

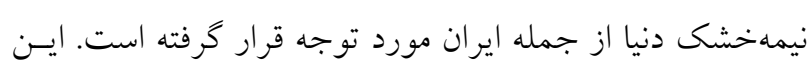

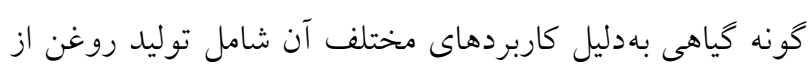

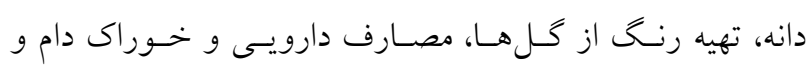

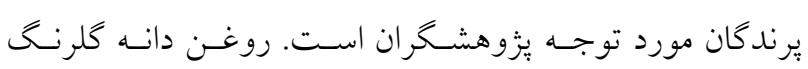

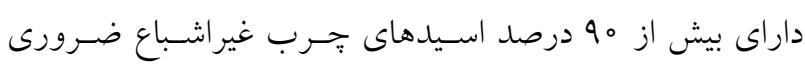

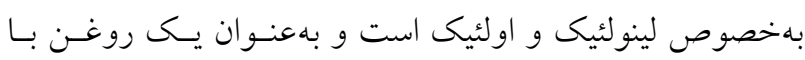
كيفيت بالا محسوب مى شود (4) (ب). خشكى يكى از تنشهاى محيطى است كه به بعنوان مهم ترين

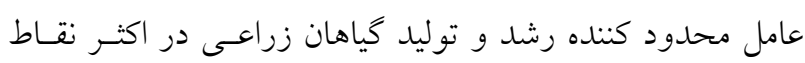

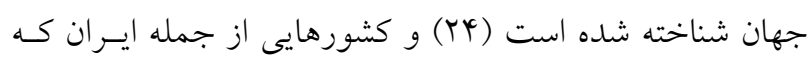

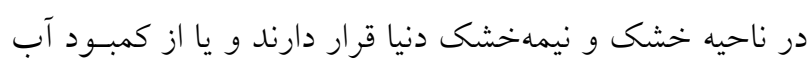

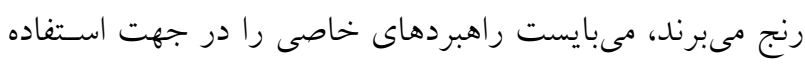

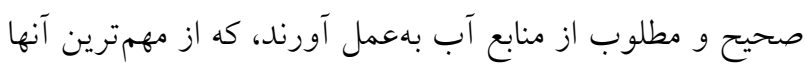
توليد ارقام متحمل به كم آبى و با عملكرد بالا است.

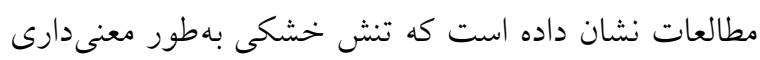

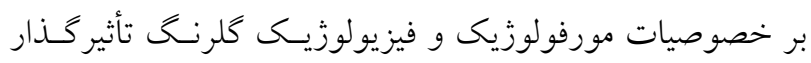
بوده و درنهايت موجب كاهش معنى دار عملكرد دانسه مسى شـود

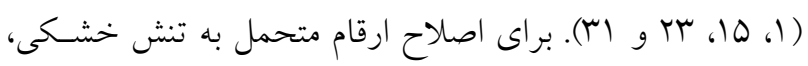

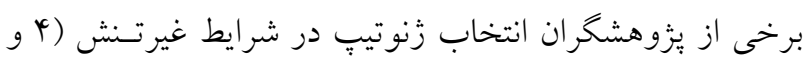

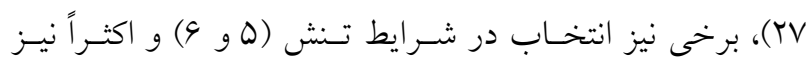

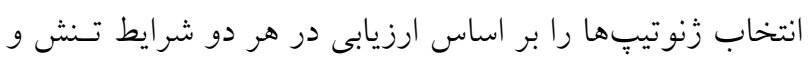

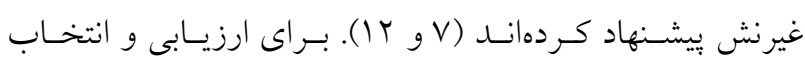

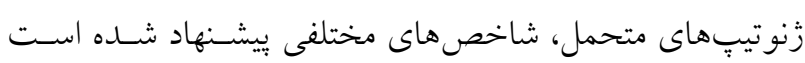

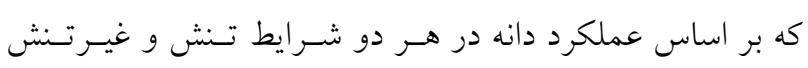

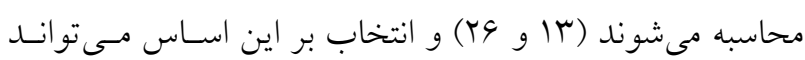

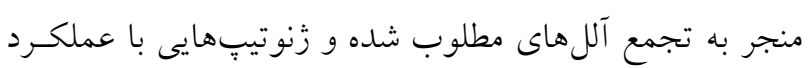
بالاتر براى هر دو محيط انتخاب شوند (YN). 
(سانتى متر) و B جُخالى ظاهرى خاى هستند.

براى تعيين ميزان آب مورد نياز هـر واحــــ آزمايشـى، ابتــا عمق آبيارى از معادله ب محاسبه شد:

$\mathrm{I}=\left(\theta_{\mathrm{FC}}-\theta_{\text {irri }}\right) \times \mathrm{B} \times \mathrm{D}$

در معادله فوق B جֶگالى ظاهرى خاى، D عمـق توسـعه ريشـه (سانتى متر)، $\theta_{\text {FC }}$ روبت وزنى خاى در حد ظرفيـت زراعى و 0irri

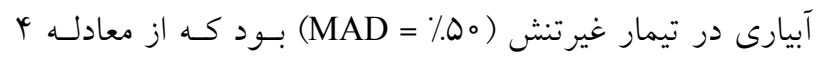

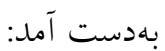

$\theta_{\text {irri }}=\theta_{\mathrm{FC}}-\left[\left(\theta_{\mathrm{FC}}-\theta_{\mathrm{PWP}}\right) \times \mathrm{MAD}\right]$

براى اندازهيرى ميزان آب وروديى به واحدهاى آزمايشى نيز از كنتور آب استفاده شد.

در پايـان آزمـايش و بـس از رسـيدكى فيزيولوزيـك، كـل بوتههاى هر واحد آزمايشى بعـد از حسذف حاشـيه برداشـت و سبس خرمنكوبى و بوجارى شد و عملكرد دانه در واحد سطح (كيلو گرم در هكتار) براى هر واحد آزمايشى محاسبه شد.

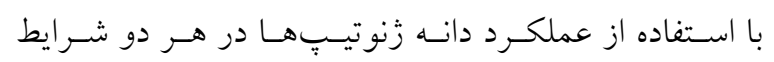

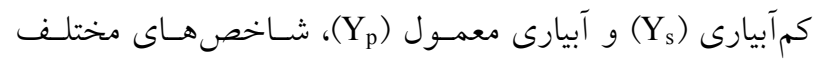
تحمل و حساسيت به تنش خشكى طبق معادلات زيـر محاسبه

$\mathrm{MP}=\frac{\mathrm{Ys}+\mathrm{Yp}}{r}$

$\mathrm{STI}=\frac{\mathrm{Y}_{\mathrm{s}} \times \mathrm{Y}_{\mathrm{p}}}{\overline{\mathrm{Y}}_{\mathrm{p}}^{Y}}$

$$
\mathrm{GMP}=\sqrt{\mathrm{Y}_{\mathrm{p}} \times \mathrm{Y}_{\mathrm{s}}}
$$

$\mathrm{DI}=\frac{\left[\mathrm{Ys} \times\left(\frac{\mathrm{Ys}}{\mathrm{Yp}}\right)\right]}{\overline{\mathrm{Y}}_{\mathrm{s}}}$

$\mathrm{M}_{\mathrm{p}} \mathrm{STI}=\frac{(\mathrm{Yp})^{r}}{(\overline{\mathrm{Y}} \mathrm{p})^{r}} \times \mathrm{STI}$

$M_{\mathrm{s}} \mathrm{STI}=\frac{(\mathrm{Ys})^{r}}{(\overline{\mathrm{Y}} \mathrm{s})^{r}} \times \mathrm{STI}$
شد. اين زنوتيقها بهطور جداكانه در دو شرايط آبيارى معمـول (غيرتنش) و كمآبيارى (تنش) بر اساس •ه و هم درصد تخليسه رطوبـت از خـاك، طـى دو سـال زراعى هوس ا و وهبا و و بـا استفاده از طرح بلوكهاى كامل تصادفى با دو تكرار در مزرعسه تحقيقاتى دانشخاه صنعتى اصفهان واقع در لورى نجـف آبـاد در

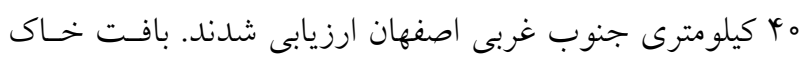

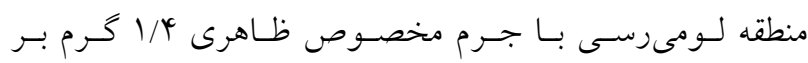

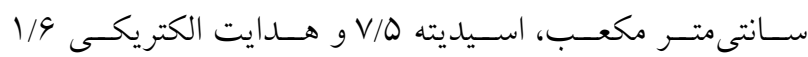
دسىزيمنس بر متر بود. هر واحد آزمايشى در اين مطالعه شامل r رديف كاشت r مترى با فاصله رديف •ه سانتى متر و فاصسله بوته در رديف برابر ه إنتى متر بود.

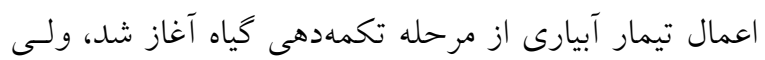
تا اين مرحله از رشد كياه، آبيارى براى دو آزمـايش در شـرايط تنش و غيرتنش يكسان انجام شد. در شرايط غيـرتنش و تسنش، بهترتيب بر اساس مها و هم درصد تخليه رطوبتى خاك، آبيـارى صورت كرفت. براى بــيشبينى مقـدار تخليسه رطـوبتى خـاك،

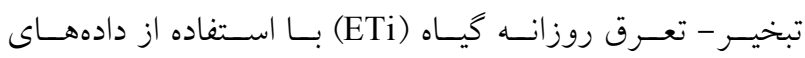
هواشناسى و رابطه بِنمن - مانتيث (Penman-Monteith) (معادله () بهشرح زير محاسبه شد (r):

$\mathrm{ET}_{\mathrm{i}}=\mathrm{ET}_{0} \times \mathrm{K}_{\mathrm{c}}$

در معادله فوق ET0 تبخير - تعرق يتانسيل يا مرجع (بـر اسـاس

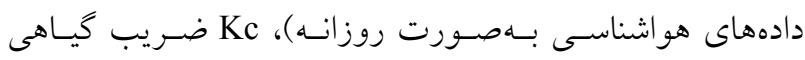
كلرنگ و ETi تبخير و تعرق روزانه هستند. در ضمن زمانى كه مقدار تبخير - تعرق تجمعى در طى رشد

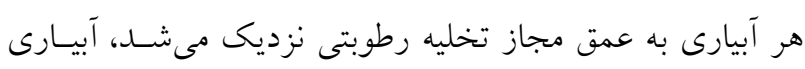
انجام مى گرفت و عمق مجاز تخليه رطـوبتى نيـز بــا اسـتفاده از معادله Y محاسبه شد:

$\mathrm{RAW}=\left(\theta_{\mathrm{FC}}-\theta_{\mathrm{PWP}}\right) \times \mathrm{MAD} \times \mathrm{D} \times \mathrm{B}$

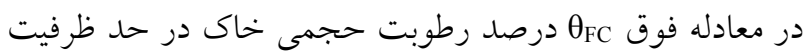
زراعى، دائم، MAD ضريب مديريت مزرعه (0ه و هم درصد بهترتيـب در تيمار غيـرتسنش و تسنش رطـوبتى)، D عمـق توسـعه ريشـه 


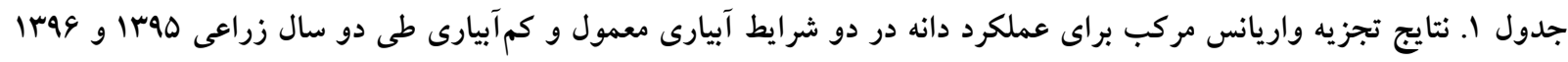

\begin{tabular}{|c|c|c|}
\hline ميانكين مربعات & درجه آزادى & منابع تغيير \\
\hline rOVYVVV** & 1 & سال \\
\hline$|r| 90 \Delta V Y \varphi^{* *}$ & 1 & شرايط آبيارى \\
\hline$V M M^{n s}$ & 1 & سال × شرايط آبيارى \\
\hline $91 \cdot \wedge 9$ & $r$ & تكرار / سال × شرايط آبيارى \\
\hline $111 \mu \circ V^{* *}$ & ro & رنوتيب \\
\hline roVarV** & ro & زنوتيب × سال \\
\hline $9 \circ 9 \circ 40^{\mathrm{ns}}$ & ro & زنوتيب × شرايط آبيارى \\
\hline$\left|10 \mu_{0}\right|^{* *}$ & ro & زنوتيٍ × سال × شرايط آبيارى \\
\hline DQ194 & $\wedge \circ$ & اشتباه آزمايشى \\
\hline
\end{tabular}

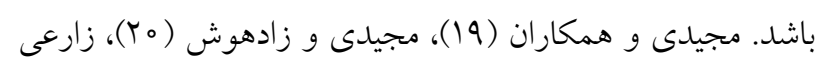

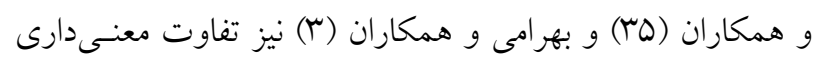

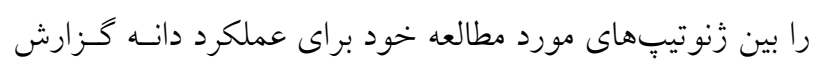

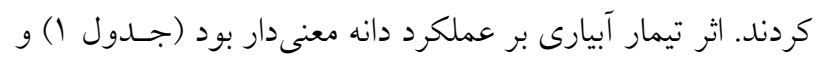
كاهش عملكرد دانه در محيط تنش مشاهده شد. كـاهش عملكـرد

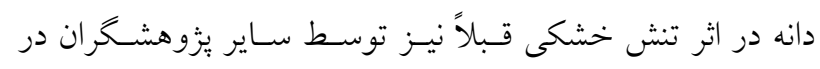

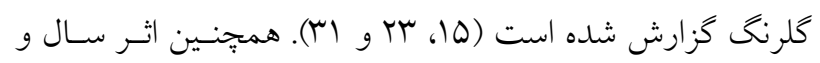

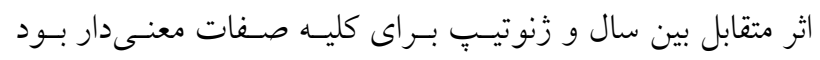

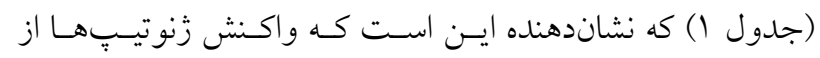

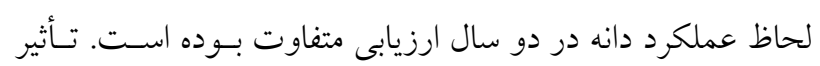

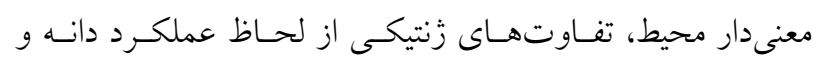

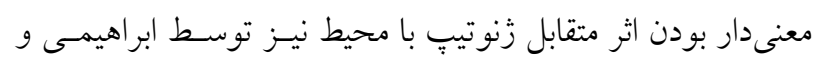
همكاران (N) در گلرنخ كزارش شده است.

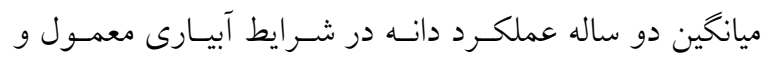

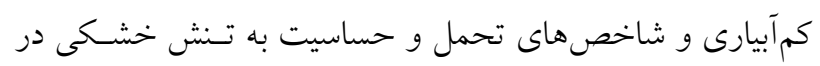
جدول r آورده شده است. بيشترين و كمترين مقدار عملكرد دان دانه

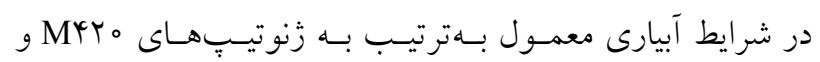

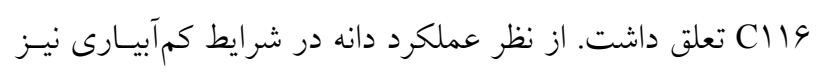

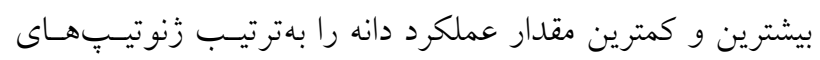

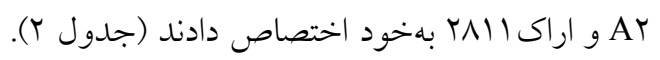

SNPI $=\sqrt[r]{(Y p+Y s) /(Y p-Y s)} \times \sqrt[r]{Y p \times Y s \times Y s}$

$\mathrm{SDI}=\frac{\mathrm{Yp}-\mathrm{Ys}}{\overline{\mathrm{Y}} \mathrm{p}}$

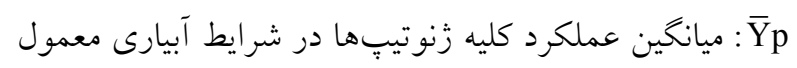
ميانخين عملكرد كليه زنوتيبها در شرايط كم آبيارى :

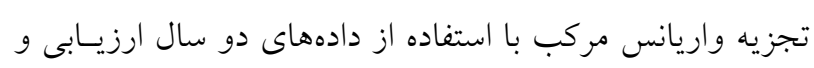
در قالب طرح بلوكهاى كامل تصادفى بــا استـفاده از نــرمافـزار SAS

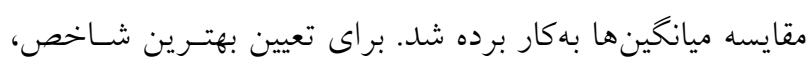

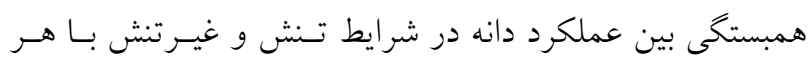

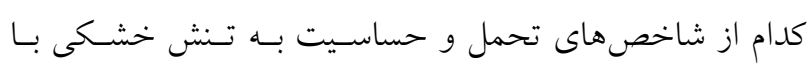

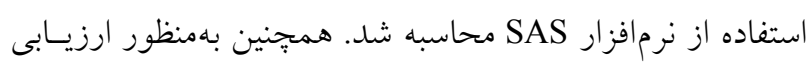

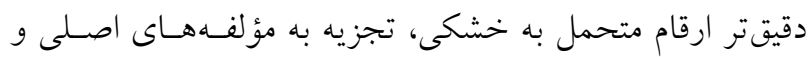

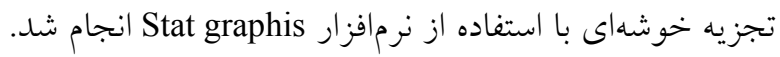

\section{نتايج و بحث}

نتايج تجزيه واريانس نشان داد كه زنوتيِهاى مـورد برررسى از

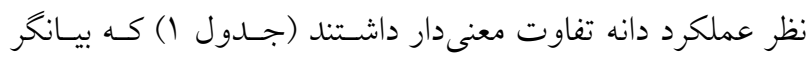

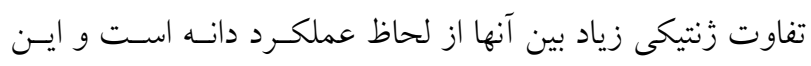
تنوع مىتواند در جهت بهبود آنها توسط روشهاى نهاى انتخاب مـؤثر 
جدول Y. ميانكين عملكرد دانه (كيلوگرم در هكتار) در شرايط آبيارى معمول (Y) و كمآبيارى (Y) در دو سال آزمايش و شاخصهاى تحمل و حساسيت به تنش خشكى

\begin{tabular}{|c|c|c|c|c|c|c|c|c|c|c|}
\hline $\mathrm{M}_{\mathrm{s}} \mathrm{STI}$ & $\mathrm{M}_{\mathrm{p}} \mathrm{STI}$ & SDI & SNPI & DI & GMP & $\mathrm{MP}$ & STI & $\mathrm{Y}_{\mathrm{s}}$ & $Y_{p}$ & زَنوتيِ \\
\hline $0 / I V$ & $0 / \mu$ & $0 / 90$ & rYAq & $0 / T_{\Lambda}$ & TINI & TYYI & هـ/ & IrVT & MYvo & اراك rAll \\
\hline $0 / y^{k}$ &.$/ 0 \circ$ & $\circ / D Y$ & rapr & $0 / 49$ & ryqA & rA口r & $0 / 49$ & IGVY & rame & كوسه \\
\hline$\circ / 0 \circ$ &.$/ 01$ & $0 / \Psi q$ & 。וس ו & . $/ Q T$ & rGOT & $r \wedge \circ Y$ &.$/ 01$ & 1949 & revg & Al \\
\hline $1 / N r$ & $0 / 91$ & $\circ / r_{0}$ & $11 \% \circ 99$ & $1 / 1 \wedge$ & TYAq & סצM & $\circ / V q$ & $r q \circ 9$ & rVAD & Ar \\
\hline.$/ 09$ & ه & $0 / T V$ & $Y Y \circ V$ & $\circ / v q$ & TOIA & TOG & $0 / 4 V$ & TITV & r991 & $\mathrm{C} 111$ \\
\hline$\circ / \Lambda$ & $0 / 19$ & $0 / 4 Q$ & TOTV & $0 / 41$ & 1999 & ro99 & $0 / \mu$ & IKVO & TVTr & $\mathrm{C} 119$ \\
\hline $0 / 49$ & $0 / \mu 1$ & $0 / 7 q$ & FVV & $\circ / V Q$ & YYASI & TOYA & $0 /$ kq & T०YA & r०YA & $C|Y|$ \\
\hline $0 / 99$ & $0 / 4 \wedge$ & $\circ / \mu V$ & 4199 & $\circ / V^{4}$ & TSVD & TAYY & - $/ Q T$ & $r \circ \wedge l$ & TOSV & CIYA \\
\hline سח/ם & $0 / 90$ &.$/ 09$ & TVGY & هـ/ & rOV。 & rAVI & $0 / 4 \Lambda$ & $19 \mu \mathrm{V}$ & 4109 & $C+110$ \\
\hline $0 / 99$ & $1 / \circ \mu$ & $\circ / O V$ & rוس & $0 / 4 y$ & r.rr & ח & $0 / 94$ & $199^{\circ}$ & 4909 & ETYIV \\
\hline$\circ / \Delta \Lambda$ & $0 / \pi q$ & $0 / Y \Lambda$ & GEYO & $\circ / V q$ & TYVG & TOY。 & $0 / 40$ & rITO & rq10 & ETYTV \\
\hline $1 / 19$ & $\circ / \Lambda \mid$ & $\circ / Y_{0}$ & $\Delta \varphi \circ \Delta$ & . & r०Yl & TrVo & $0 / 9 \mathrm{~V}$ & TrG. & fivg & ETYYA \\
\hline$\circ \mu \circ$ & OMY &.$/ Q F$ & rVTl & $\circ / \mu \wedge$ & TYOY & roqY & O/KY & $19 \mu$ & 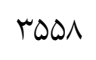 & HrV \\
\hline$\circ / \wedge \Delta$ & ०/VG & $0 / 40$ & 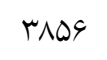 & $0 / 9 Y$ & ra91 & MIT。 & $0 / 94$ & TMIT & $r \circ r q$ & KIT \\
\hline $0 / 91$ & $\circ / N$ & $0 / \mu \wedge$ & $Y Y \wedge$ O & o/VG & rasV & r.q1 & $0 / 94$ & $r \mu \circ \mid$ & TMN & KYI \\
\hline $1 / Y^{K}$ & $1 / \pi V$ & $\circ / 4 \wedge$ & $r \circ \Lambda Y$ & $0 / 90$ & A MAN & rosI & O/AY & TKYO & FVor & MYto \\
\hline$\circ / V Y$ & - /Qr & $0 / 49$ & $\varphi \circ \Delta \wedge$ & $\circ / N$ & TVVG & rAVr & $\circ / 09$ & TrII & roro & SIYY \\
\hline $1 / 00$ & $0 / 94$ & $0 /$ ky & pooq & $0 / 91$ & r. & TrVI & $\circ / V \circ$ & TYQV & EYTE & SIYq \\
\hline $0 / \mu q$ & سח/ם & $0 / M Y$ & VA9T & $0 / 99$ & TMVD & TOQS & $0 / 41$ & IMAV & Mrtu & STIl。 \\
\hline $0 / 9 \mu$ & $\circ / \mathrm{VV}$ & - & l. & $\circ / 0 \circ$ & rNMK & Mor &.$/ 09$ & 1919 & prat & AC-Stirling \\
\hline $0 / Y \Lambda$ & $0 / 41$ &.$/ 01$ & rVAI & $0 / 41$ & Yイו & rDSV & $0 / 41$ & larv & ryqa & AC-Suncet \\
\hline
\end{tabular}

STI :شاخص تحمل به تنش، MP: ميانخين توليد، GMP: ميانخين هندسى توليد، DI: شاخص مقاومت به خشكى، SDI : شاخص ميزان محصول محيط غيرتنش

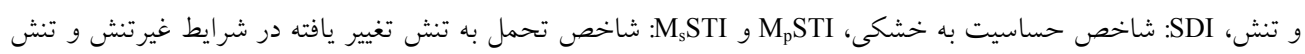

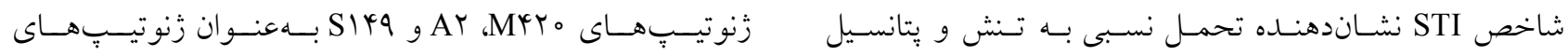

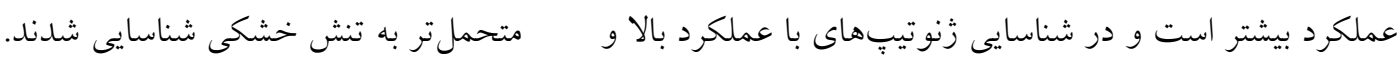

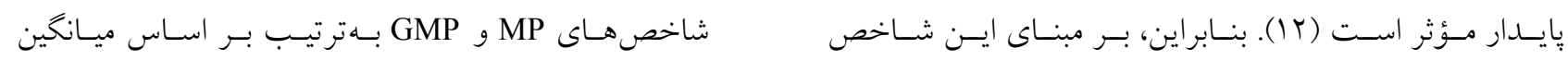

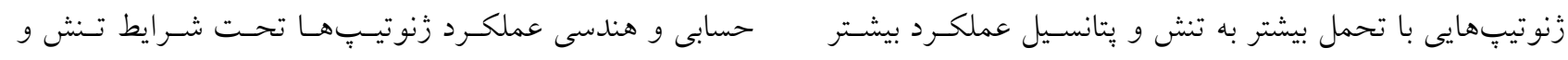

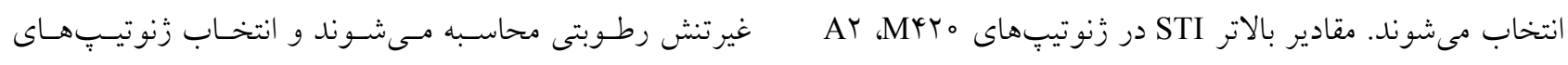

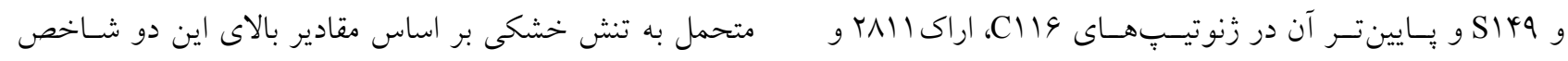

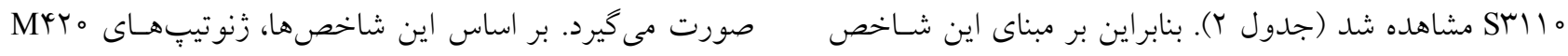




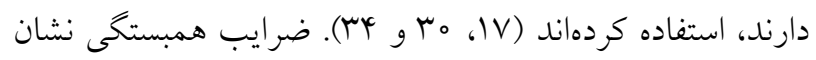
داد كـه شـاخصهــاى STI، MPSTI GMP ، و MPSTI ،

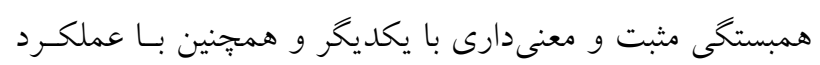

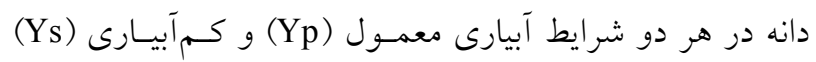

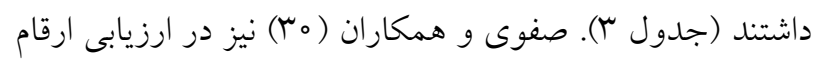

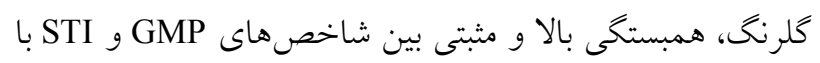

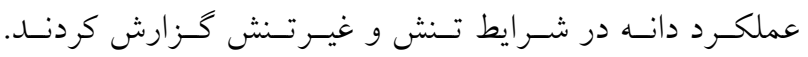
همجنين، يارى و همكاران (Tr) مشاهده كردند كه شاخصهاى MP و Mر شرايط تنش خشكى در مرحلـه GMP، STI ،SNPI

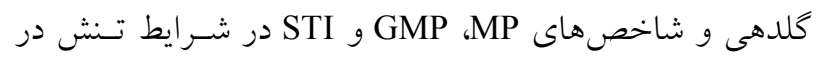
مرحله دانهبندى براى شناسايى ارقـام كُلزنـع بـا عملكـرد بـالا

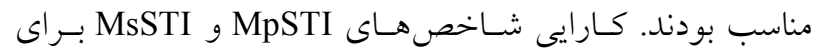

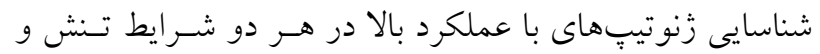
غيرتنش خشكى نيز توسط ساير يزوهشكران در گياهان مختلف

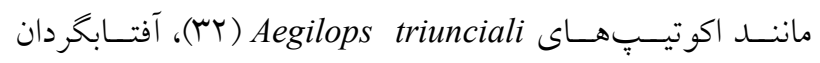

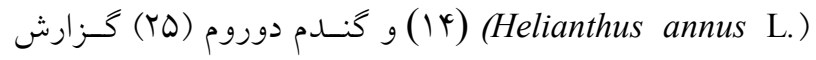

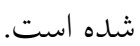
شاخصهاى DI و SNPI با عملكرد دانهه در شـرايط تسنش

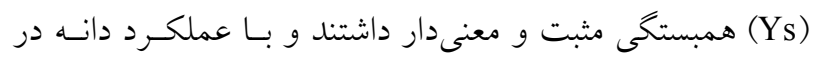

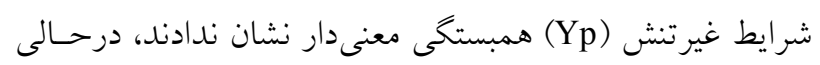

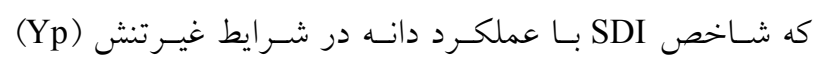

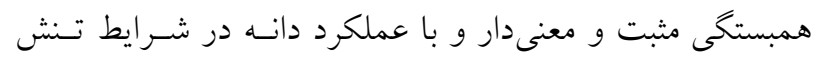

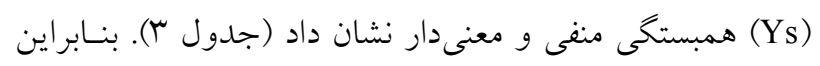

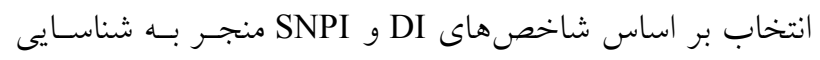

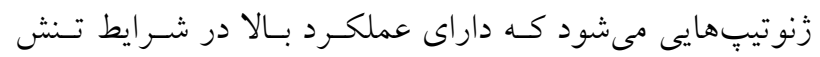
هستند و انتخـاب بـر اسـاس شـاخص SDI، زنوتيـٍِهـايى بـا

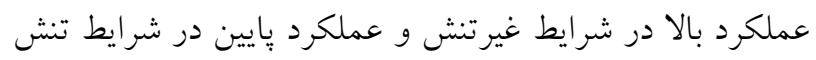
را شناسايى مى كند. بلهطور كلى شاخصى مناسـب اسـت كـه در هـر دو شـرايط غير تنش و تنش همبستكى مثبت و معنىدارى با عملكرد داشـه

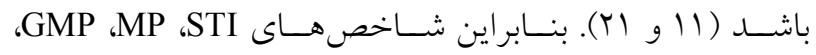
MsSTI و بهدليل دارا بودن همبستخى مثبت و معنىدار

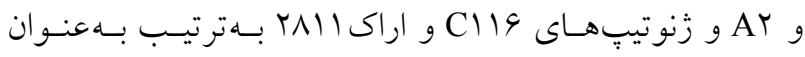

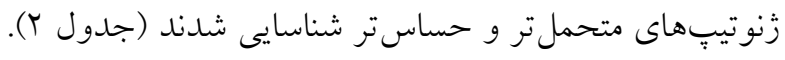

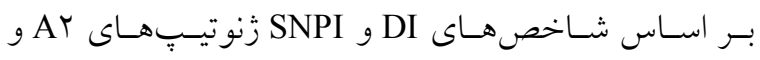

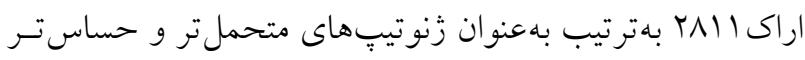

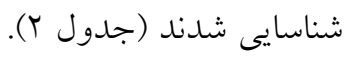
مقدار بالاى شاخص SDI، نشانه حساسيت زنوتيبٍ به تنش

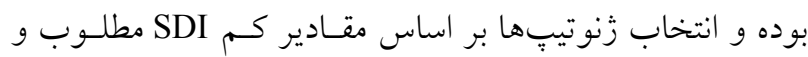

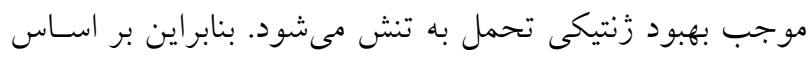

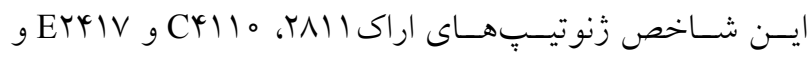

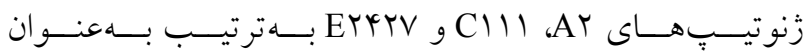

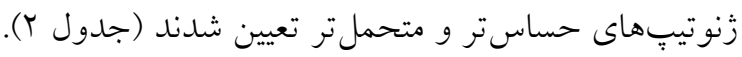
شاخص هاى MpSTI و MsSTI همانند شاخص STI بـوده و زنوتيبهاى با مقادير بــالى ايسـن شـاخصهـا، بـراى هـر دو

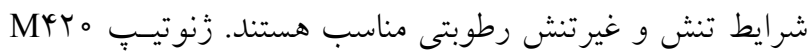
كه در دو شرايط تنش و غيرتنش رطوبتى عملكرد بالايى داشـته است، مقدار بالاى هر دو شـاخص MpSTI و MsSTI را نشـان

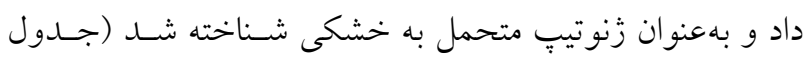

انتخاب بر اساس شاخصهاى MP و GMP باعث گـزينش

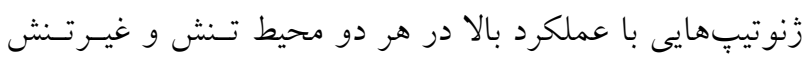

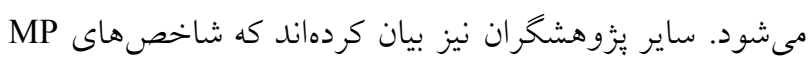

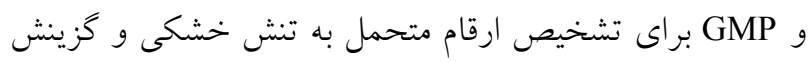

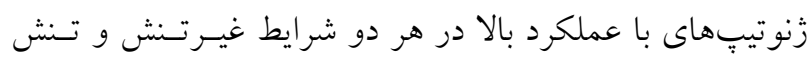

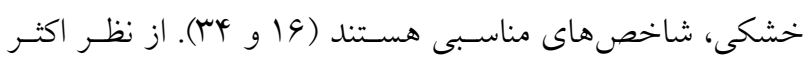

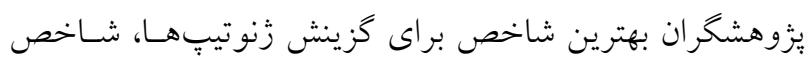

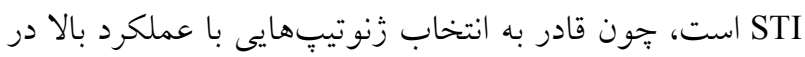

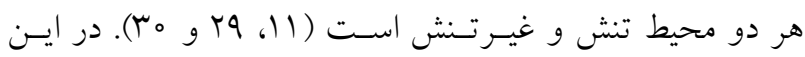

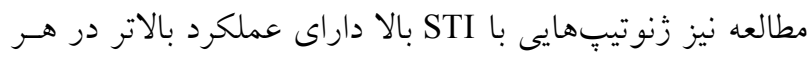
دو شرايط تنش و غيرتنش بودند.

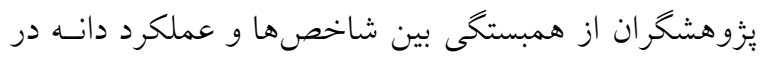
شرايط تنش و غيرتنش نيز براى انتخاب بهترين شاخصهـا كـهـ

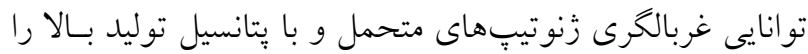




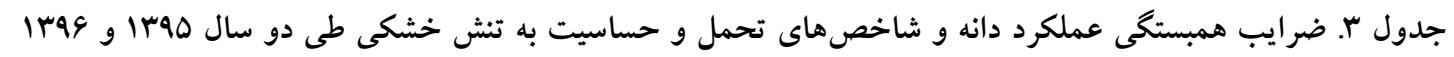

\begin{tabular}{|c|c|c|c|c|c|c|c|c|c|c|}
\hline $\mathrm{M}_{\mathrm{s}} \mathrm{STI}$ & $\mathrm{M}_{\mathrm{p}} \mathrm{STI}$ & SDI & SNPI & DI & GMP & MP & STI & $\mathrm{Y}_{\mathrm{s}}$ & $Y_{p}$ & صفات \\
\hline & & & & & & & & & 1 & $\mathrm{Y}_{\mathrm{p}}$ \\
\hline & & & & & & & & 1 & $0 / M Y^{n s}$ & $\mathrm{Ys}_{\mathrm{s}}$ \\
\hline & & & & & & & 1 & $\circ / \Lambda \Delta^{* *}$ & $\circ / V \varphi^{* *}$ & STI \\
\hline & & & & & & 1 & $\circ / 9 V^{* *}$ & $\circ / Y^{* *}$ & $\circ / \wedge 9^{* *}$ & MP \\
\hline & & & & & 1 & $\circ / 9 V^{* *}$ & $\circ / 99^{* *}$ & $\circ / \Lambda r^{* *}$ & $\circ / V^{* *}$ & GMP \\
\hline & & & & 1 & $\circ / \Delta r^{*}$ & $\circ / \mathrm{V}^{\mathrm{ns}}$ & $\circ / D Y^{*}$ & $\circ / 90^{* *}$ & $-0 / \circ q^{n s}$ & DI \\
\hline & & & 1 & $\circ / \wedge r^{* *}$ & $0 / \mu Y^{n s}$ & $0 / 4 T^{n s}$ & $\circ / 4 y^{*}$ & $\circ / N I^{* *}$ & $-0 / 0 \mu^{\mathrm{ns}}$ & SNPI \\
\hline & & 1 & $-\circ / V_{0}^{* *}$ & $-0 / 91^{* *}$ & $-\circ / / V^{n s}$ & $\circ / \circ Y^{n s}$ & $-\circ / \mathrm{IVns}$ & $-0 / 9 V^{* *}$ & $0 / \mathbb{F} V^{*}$ & SDI \\
\hline & 1 & $\circ / 1 Q^{\mathrm{ns}}$ & $\circ / Y I^{\mathrm{ns}}$ & $\circ / Y^{n s}$ & $\circ / 9 r^{* *}$ & $\circ / 99^{* *}$ & $\circ / 9 r^{* * *}$ & $\circ /\left.9\right|^{* *}$ & $\circ / 91^{* *}$ & $\mathrm{M}_{\mathrm{p}} \mathrm{STI}$ \\
\hline 1 & $\circ / V Q^{* *}$ & $-0 / 4 \varphi^{*}$ & $0 / 99^{* *}$ & $\circ / \mathrm{V} \mathrm{V}^{* *}$ & $\circ / 91^{* *}$ & $\circ / \Lambda \mu^{* *}$ & $\circ / 9 r^{* *}$ & $0 / 9 r^{* *}$ & $\circ / 00^{*}$ & $\mathrm{M}_{\mathrm{S}} \mathrm{STI}$ \\
\hline
\end{tabular}

و و شاخص SDI و همبستخى منفى با شاخص هـLاى SNPI و DI داشت (جدول \&). بنابراين انتخاب بر اسـاس مقـادير بيشـتر

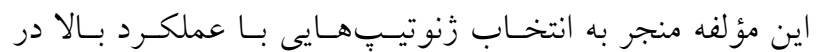

$$
\text { شرايط غيرتنش مىشود. }
$$

بر اساس باى يلات ترسيم شده و بر مبناى مؤلفههاى اصسلى

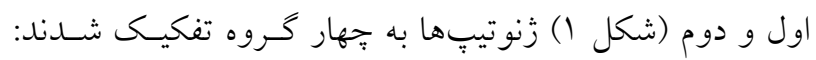
كروه اول شامل زنوتيتٍ AT بود كه بيشترين عملكرد در شرايط تنش و شاخص هاى SNPI MSSTI و DI و كمتـرين شـاخص MP را داشت. گروه دوم نيز مقادير بـالاى شـاخصهـاى SDI

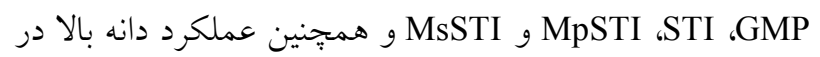
هر دو شرايط تنش و غيـرتنش را داشـتند و ايسن خـروه شـامل

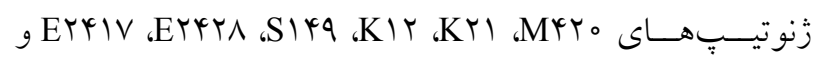
AC-Stirling بودند. بنابراين، اين دو گرووه بهعنـوان پِيـــارترين زنوتيِها از نظر عملكرد دانه در دو تيمار آبيارى شناخته شدند و بــراى هـر دو محسيط تسنش و غيـرتنش بيشـنهاد مسىشـوند. زنوتيـتهــاى كروه سوم را تشكيل دادند كه مقدار بالاى شـاخصهـاى SNPI و DI و مقدار بايين شاخص SDI را بهذــود اختصـاص دادنـد.
با عملكرد دانه در هر دو شـرايط غيـرتنش و تـنش (جـــول ؟) بهنوان بهترين شاخص ها براى شناسايى زنوتيٍِهاى متحملتر و با عملكرد دانه بيشتر براى هـر دو تيمـار آبيـارى در كلرنـح

شناخته شدند. بهمنظور درك بيشتر ارتباط بين شاخص ها و عملكرد دانسه و كروهبندى زنوتيِها از تجزيه بـهـ مؤلفـهــاى اصـلى و ترسـيم كرافيكى باىيلات و تجزيه خوشـهاى بـهروش Ward اسـتفاده شد (شكل هاى ( و Y). دو مؤلفه اصلى اول جمعاً $90 / 9$ درصــ

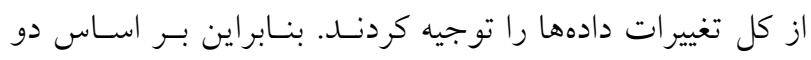
مؤلفه اول باى يلات ترسيم شد. اولين مؤلفه 94/0 درصد از كـل تغييرات دادهها را توجيه كرد و با Ys، Yp و شاخصهـاى MP، GNPI و MsSTI ،MPSTI ،STI ،GMP بالايى داشت (جدول \&). بنابراين، اين مؤلفه را مسىتـوان مؤلفـه. يايدارى عملكرد و تحمل به خشكى نامخذارى كرد. انتخاب بـر اساس مقادير بالاتر اين مؤلفه، زنوتيبٍهايى را گزينش مسىكنـد كه تحمل بيشترى به تنش خشكى داشـته و عملكــرد بـالايى در هر دو شرايط تنش و غيرتنش دارند. دومين مؤلفـه مقـدار درصد از كل تغييرات دادهها را توجيه كرد و همبستخى مثبت با 


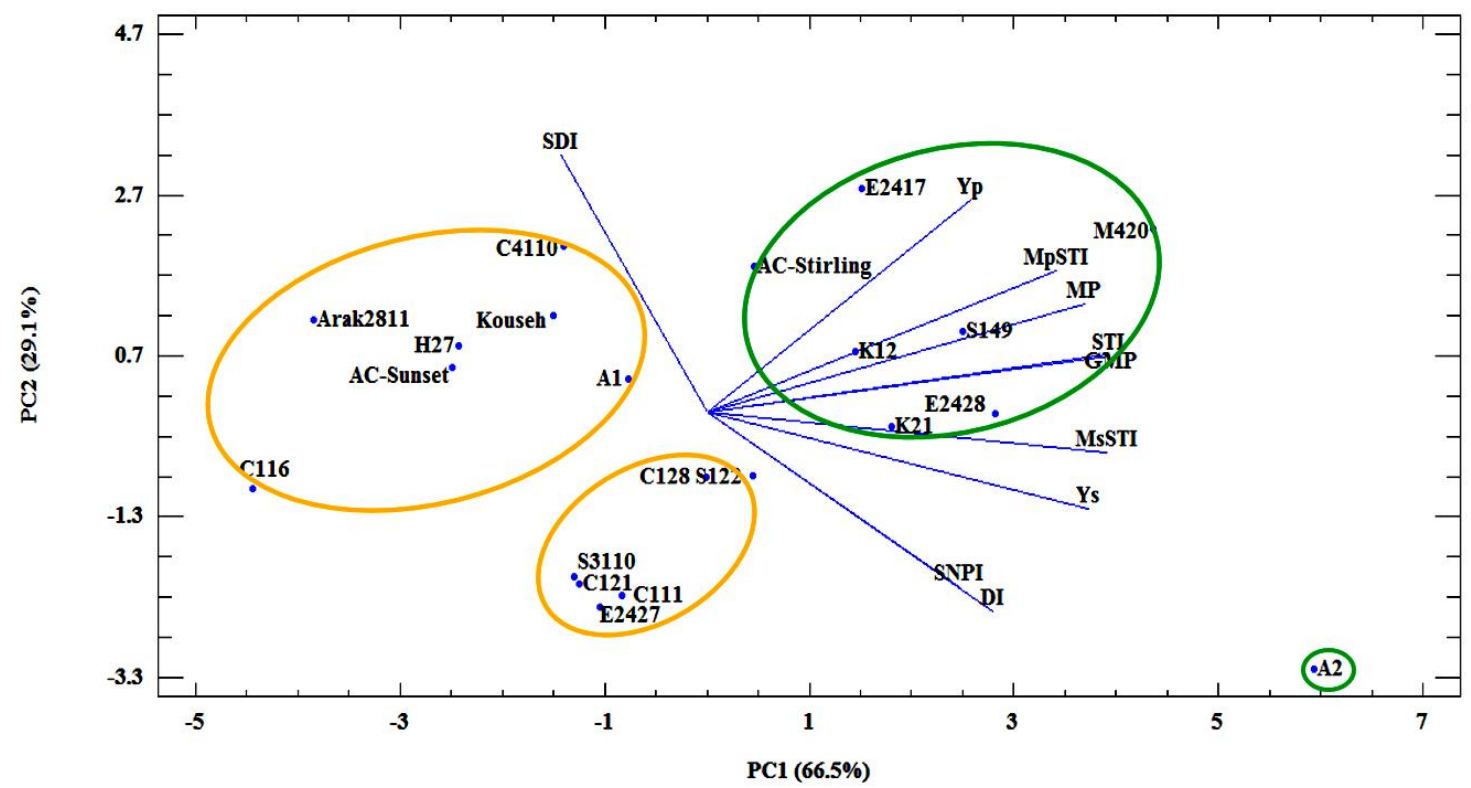

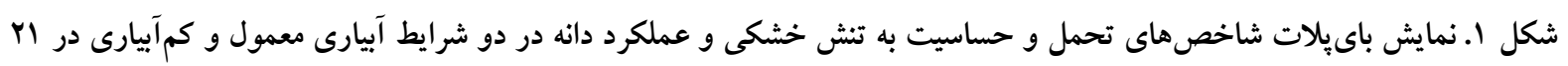

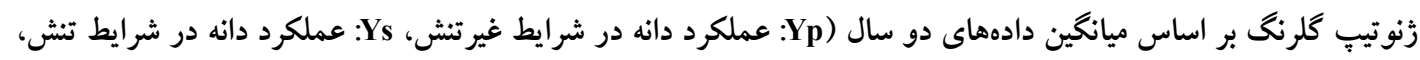

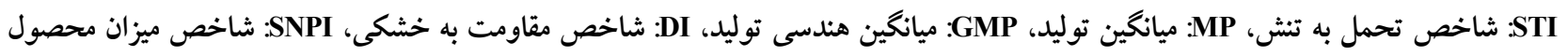
محيط غيرتنش و تنش، SDI: شاخص حساسيت به خشكى، MPSTI و MsSTI: شاخص تحمل به تنش تغييريافته در شرايط غيرتنش و تنش)

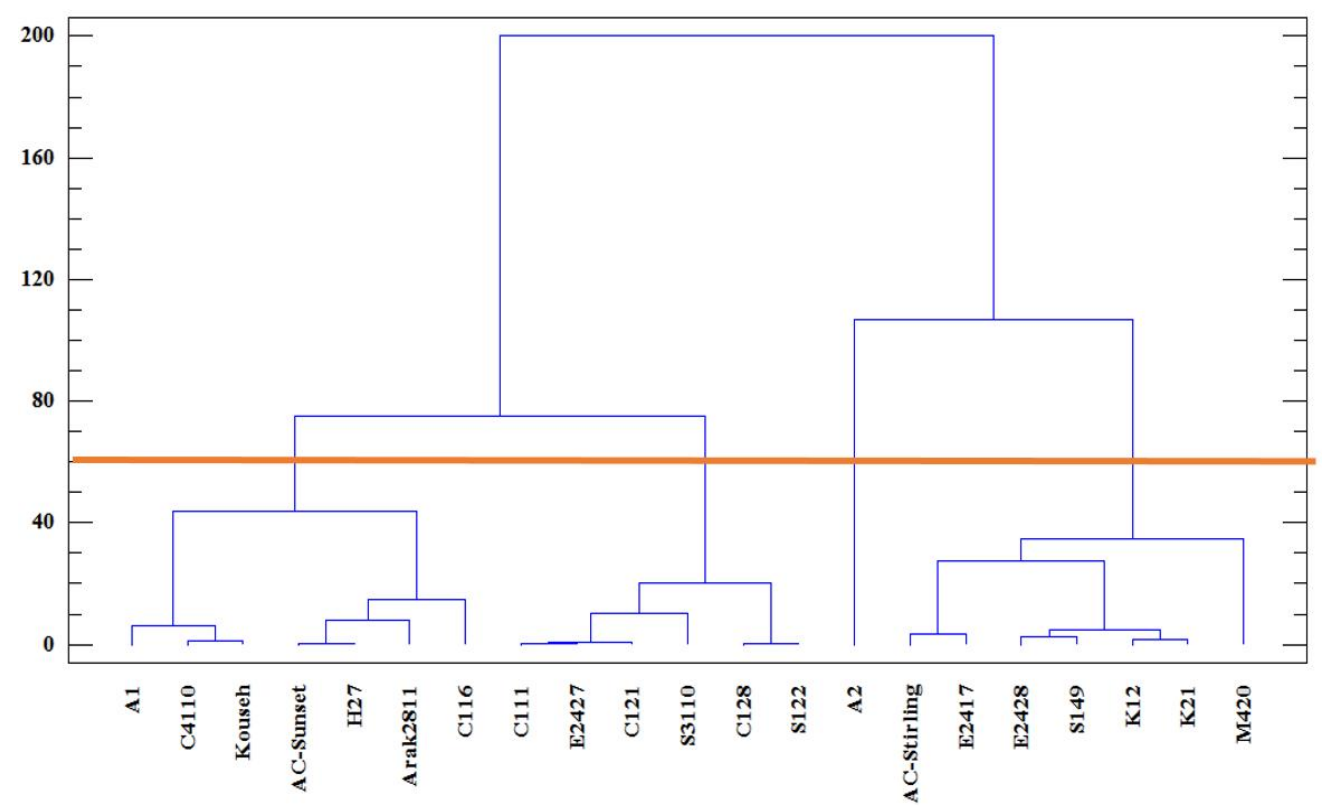

شكل r. نمودار درختى تجزيه خوشهاى بر اساس شاخصهاى تحمل و حساسيت به تنش خشكى و عملكرد دانه در دو شرايط آبيارى معمول و كم آبيارى طى دو سال

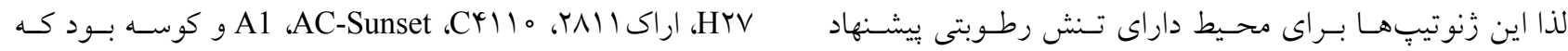

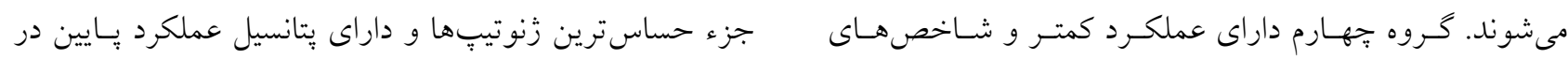

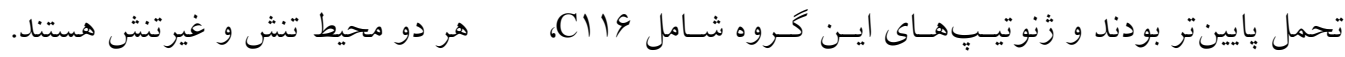


جدول fا. درصد تنوع هر مؤلفه و ضرايب عملكرد دانه و شاخص هاى تحمل و حساسيت به تنش خشكى

\begin{tabular}{|c|c|c|c|c|c|c|c|c|c|c|c|}
\hline $\mathrm{M}_{\mathrm{s}} \mathrm{STI}$ & $\mathrm{M}_{\mathrm{p}} \mathrm{STI}$ & SDI & SNPI & DI & GMP & MP & STI & Ys & $Y_{p}$ & درصد تنوع & مؤلفهها \\
\hline.$/ 9 V$ & $\circ / \wedge Q$ & $-0 / \mu^{4}$ & $0 / 91$ & $0 / 99$ & $\circ / 9 V$ & ०/9r &.$/ 91$ &.$/ 94$ & $0 / 94$ & $99 / 0$ & مؤلفه اول \\
\hline$-0 / 14$ & $\circ / \Delta \circ$ & $0 / 91$ & $-0 / 94$ & $-0 / V 1$ & $0 / T_{1}$ & $\circ / \Lambda$ & $0 / Y_{0}$ & -o/r山 & $\circ / V \Delta$ & $r q / 1$ & مؤلفه دوم \\
\hline
\end{tabular}

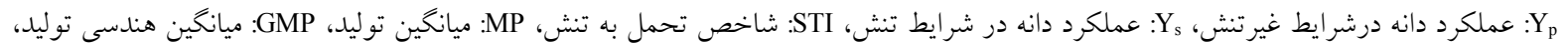

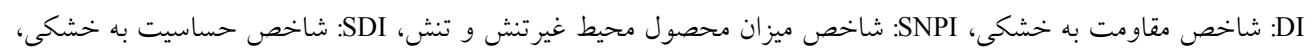
M و M M STI

MpSTI ،MP ، MP ، MPTI

برتر براى هر دو تيمار آبيارى مناسبتر شناخته شدند. بـر اسـاس

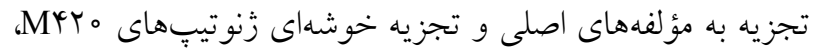

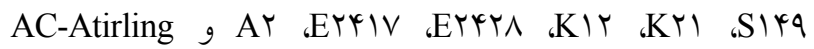

بهعنو ان زنوتيِّهاى متحمل تر و با يتانسيل عملكرد مطلوب بـر اي

هر دو شرايط تنش و غيرتنش تعيين شدند و از ايسن زنوتيـيٌهـا

$$
\text { مىتوان در برنامههاى اصلاحى كلرنخ استفاده كرد. }
$$

$$
\begin{aligned}
& \text { نمودار درختى حاصل از تجزيه خوشهاى در شكل ب ارائه شــه } \\
& \text { است كه زنوتيبٍ ها به جهار خوشه تقسيم شدند. نتـايج حاصل } \\
& \text { از تجزيه خوشهاى مشابه نتايج حاصل از تجزيه بــه مؤلفـهــاى } \\
& \text { اصلى بودند و تأييد كننده آن نتايج هستند. } \\
& \text { در مجموع نتايج اين مطالعه نشان داد كـهـ بـين زنوتيـٍِهـاى } \\
& \text { مورد مطالعه، تفاوت معنى دار از نظر عملكرد دانسه وجــود داشـت } \\
& \text { كه از اين تنوع مىتوان در برنامههاى انتخــاب بــراى توليــ ارقـام } \\
& \text { اصلاح شده با عملكرد بالا استفاده كرد. در ضـمن شـاخص هــاى }
\end{aligned}
$$

منابع مورد استفاده

1. Abd El-Lattife, E. A. 2013. Safflower yields and water use efficiency as effected by irrigation at different soil moisture depletion levels and plant population density under arid conditions. Archives of Agronomy and Soil Science 59: 1545-1557.

2. Allen, R. G., L. S. Pereira, D. Raes and M. Smith. 1998. Crop Evapotranspiration-Guidelines for Computing Crop Water Requirements. In: FAO Irrigation and Drainage Paper. No. 56. FAO, Rome.

3. Bahrami, F., A. Arzani and V. Karimi. 2014. Evaluation of yield-based drought tolerance indices for screening safflower genotypes. Agronomy, Soils \& Environmental Quality 106: 1219-1224.

4. Betran, F. J., D. Beck, M. Banziger and G. O. Edmeades. 2003. Genetic analysis of inbred and hybrid grain yield under stress and non-stress environments in tropical maize. Crop Science 43: 807-817.

5. Blum, A. 1996. Crop responses to drought and the interpretation of adaptation. Plant Growth Regulation 20: 135-148.

6. Ceccarelli, S. and S. Grando. 1991. Selection environment and environmental sensitivity in barley. Euphytica 57: 157-167.

7. Clarke, J. M., R. M. De-Pauw and T. M. Townley-Smith. 1992. Evaluation of methods for quantification of drought tolerance in wheat. Crop Science 32: 728-732.

8. Ebrahimi, F., M. M. Majidi, A. Arzani and Gh. Mohammadi-negad. 2016. Oil and seed yield stability in a worldwide collection of safflower under arid environments of Iran. Euphytica 212: 131-144.

9. Farshadfar, E. and J. Sutka. 2002. Screening drought tolerance criteria in maize. Acta Agronomuca Hungarica 50: 411416.

10. Farshadfar, E. and J. Javadinia. 2011. Evaluation of Chikpea (Cicer arietinum L.) genotypes for drought tolerance. Seed and Plant Improvement Journal 27: 517-537.

11. Farshadfar, E., M. Saeidi and S. J. Honarmand. 2012. Evaluation of drought tolerance screening techniques among some landraces of bread wheat genotypes. European Journal of Experimental Biology 2: 1585-1592.

12. Fernandez, G. C. J. 1992. Effective selection criteria for assessing plant stress tolerance. Proceedings of the International Symposium on Adaptation of Vegetables and other Food Crops to Temperature and Water Stress. AVRDC, Shanhua, Taiwan13-18 Aug, 1992.

13. Fischer, R. A. and R. Maurer. 1978. Drought resistance in spring wheat cultivars. Part 1: grain yield response. 
Australian Journal of Agriculture Research 29: 897-912.

14. Gholinezhad, E., R. Darvishzadeh and I. Bernousi. 2014. Evaluation of drought tolerance indices for selection of confectioney sunflower (Helianthus annus L.) landraces under various environmental conditions. Notulae Botanicae Horti Agrobotanici Cluj-Napoca 42: 187-201.

15. Janmohammadi, M., N. Mohammadi, F. Shekari, A. Abbasi and M. Esmailpour. 2017. The effects of silicon and titanium on safflower (Carthamus tinctorius L.) growth under moisture deficit condition. Acta Agriculturae Slovenica 109: 443-455.

16. Khalili, M., A. Pour-Aboughadereh, M. R. Naghavi and E. Mohammad-Amini. 2014. Evaluation of drought tolerance in safflower genotypes based on drought tolerance indices. Notulae Botanicae Horti Agrobotanici ClujNapoca 42: 214-218.

17. Khalili, M., A. Pour-Aboughadareh and M. R. Naghavi. 2016. Assessment of drought tolerance in barley: integrated selection criterion and drought tolerance indices. Environmental and Experimental Biology 14: 33-41.

18. Lan, J. 1998. Comparison of evaluating methods for agronomic drought resistance in crop. Acta Agriculturae Boreali-occidentalis Sinica 7:85-87.

19. Majidi, M. M., V. Tavakoli, A. Mirlohi and M. R. Sabzalian. 2011. Wild safflower species (Carthamus oxyacanthus Bieb.): a possible source of drought tolerance for arid environments. Australian Journal of Crop Science 5: $1055-1063$.

20. Majidi, M. M. and S. Zadhoush. 2014. Molecular and morphological variation in a worldwide collection of safflower under arid environments of Iran. Crop Science 54: 2109-2119.

21. Mitra, J. 2001. Genetics and genetic improvement of drought resistance in crop plants. Current Science 80: 758-763.

22. Moosavi, S. S., B. Yazdi Samadi, M. R. Naghavi, A. A. Zali, H. Dashti and A. Pourshahbazi. 2008. Introduction of new indices to identify relative drought tolerance and resistance in wheat genotypes. Desert 12: 165-178.

23. Movahhedy-Dehnavy, M., S. A. M. Modarres-Sanavy and A. Mokhtassi-Bidgoli. 2009. Foliar application of zinc and manganese improves seed yield and quality of safflower (Carthamus tinctorius L.) grown under water deficit stress. Industrial Crops and Products 30: 82-92.

24. Nooripoor, J. and P. Ehsanzadeh. 2012. Interrlations of some antioxidants, physiological characteristics and grain yield of sesame (Sesamum indicum L.) under different irrigation regimes. Iranian Journal of Field Crop Science 1: 81-91. (In Farsi).

25. Patel, J. M., A. S. Patel, C. R. Patel, H. M. Mamrutha, Sh. Pradeep and K. P. Pachchigar. 2019. Evaluation of selection indices in screening durum wheat genotypes combining drought tolerance and high yield potential. International Journal of Current Microbiology and Applied sciences 8: 1165-1178.

26. Pireivatlou, A. S., B. D. Masjedlou and R. T. Aliyev. 2010. Evaluation of yield potential and stress adaptive trait in wheat genotypes under post anthesis drought stress conditions. African Journal of Agricultural Research 5: 2829-2836.

27. Rajaram S. and M. Van Ginkle. 2001. Mexico, 50 years of international wheat breeding. pp. 579-604. In: Bonjean, A. P. and W. J. Angus (Eds.), the World Wheat Book: A History of Wheat Breeding. Lavoisier Publishing, Paris, France.

28. Richarde, R. A. 1996. Defining selection criteria to improve yield under drought. Plant Growth Regulation 20: 157-166.

29. Rosiele, A. A. and J. Hamblin. 1981. Theoretical aspect of selection for yield in stress and non-stress environments. Crop Science 21: 943-946.

30. Safavi, S. M., S. S. Pourdad and S. A. Safavi. 2013. Evaluation of drought tolerance in safflower (Carthamus tinctorius L.) under non stress and drought stress conditions. International Journal of Advanced Biological and Biomedical Research 1: 1086-1093.

31. Shahrokhnia, M. H. and A. R Sepaskhah. 2017. Physiologic and agronomic traits in safflower under various irrigation strategies, planting methods and nitrogen fertilization. Industrial Crops and Products 95: 126-139.

32. Taghipour, Z., R. Asghari Zakaria, N. Zare and P. Shaikhzadeh Mosadegh. 2013. The application of stress tolerance indices for evaluation of Aegilops triuncialis ecotypes to terminal drought tolerance. Journal of Crop Production 7: 79-93. (In Farsi)

33. Yari, P., A. H. Keshtkar and H. Mazaheri Laghab. 2016. Evaluation of water stress in spring safflower (Carthamus tinctorius L.) cultivars using tolerance indices in Hamadan region. Journal of Crop Breeding 8: 88-96. (In Farsi).

34. Zaheri, A. and S. Bahraminejad. 2012. Assessment of drought tolerance in oat (Avena sativa) genotypes. Annals of Biological Researh 3: 2194-2201.

35.Zareie, S., Gh. Mohammadinejad and S. Sardouie-Nassab. 2013. Screening of Iranian safflower genotypes under water deficit and normal conditions using tolerance indices. Australian Journal of Crop Science 7: 1032-1033.

36. Zeinali, E. 1999. Safflower (Characteristic, production \& utilization). Gorgan University of Agricultural Sciences and Natural Resources. Gorgan. (In Farsi). 


\title{
Evaluation of Drought Tolerance in Some Safflower (Carthamus tinctorius L.) Genotypes
}

\author{
Kh. Alizadeh Yeloojeh ${ }^{1}$ and Gh. Saeidi2 ${ }^{\star}$
}

(Received: December 26-2018; Accepted: September 30-2019)

\begin{abstract}
In order to evaluate drought tolerance of some safflower genotypes, an experiment was conducted at the Research Farm of Isfahan University of Technology in 2016 and 2017. In this study, 18 safflower genotypes selected from different local populations along with 2 exotic cultivars and one local population ( 21 genotypes) were separately evaluated at two normal (non-stress) and water deficit (stress) irrigation regimes, using a randomized complete block design with two replications. Drought tolerance and susceptible indices were calculated based on the seed yield. The results showed that there was significant differences among genotypes for seed yield. The genotypes M420 (4703 Kg/ha) and C116 (2723 $\mathrm{Kg} / \mathrm{ha}$ ) had the highest and lowest seed yield, respectively in normal irrigation condition, however, genotypes of A2 $(2906 \mathrm{Kg} / \mathrm{ha})$ and Arak2811 (1373 Kg/ha) had the highest and lowest seed yield, respectively in water deficit condition. Based on the correlation coefficients between indices and seed yield under both irrigation regimes, the indices of MP, GMP, STI, $\mathrm{M}_{\mathrm{p}}$ STI and $\mathrm{M}_{\mathrm{s}} \mathrm{STI}$ were recognized as the best ones for identification of drought tolerant genotypes in safflower. The graphic biplot and cluster analysis indicated that genotypes M420, S149, K21, K12, E2428, E2417, ACStirling and A2 were more tolerant genotypes to water stress.
\end{abstract}

Keywords: Safflower, Seed Yield, Tolerance Indices, Water Stress

1,2. Ph.D. Student and Professor, Respectively, Department of Agronomy and Plant Breeding, College of Agriculture, Isfahan University of Technology, Isfahan, Iran.

*: Corresponding Author, Email: gsaeidi@cc.iut.ac.ir 